\title{
Avaliação do usuário sobre o atendimento odontológico no Sistema Único de Saúde: uma abordagem à luz da humanização
}

\author{
User evaluation on dental care in the Unified Health System: \\ an approach from the standpoint of humanization
}

Suzely Adas Saliba Moimaz ${ }^{1}$

Arinilson Moreira Chaves Lima ${ }^{1}$

Cléa Adas Saliba Garbin ${ }^{1}$

José Eduardo Corrente ${ }^{2}$

Nemre Adas Saliba ${ }^{1}$

${ }^{1}$ Departamento de

Odontologia Infantil

e Social, Faculdade de Odontologia de Araçatuba,

Universidade Estadual Paulista (UNESP). R. José Bonifácio 1193, Vila Mendonça. 16015-050 Araçatuba São Paulo Brasil. sasaliba@foa.unesp.br ${ }^{2}$ Departamento de

Bioestatística, Instituto de

Biociências de Botucatu,

UNESP. Botucatu São Paulo Brasil.

\begin{abstract}
The scope of this study was to assess user evaluation about dental care in the Unified Health System and analyze the associations between this evaluation, sociodemographic characteristics, and aspects related to humanization of the services. It involved a cross-sectional survey with a quantitative approach, in which 461 users responded to individual interviews. The outcome variable was obtained by means of the question: "How do you consider the care given by the dentist and by the team in this health unit?" Responses were grouped into "positive evaluation" and "negative evaluation." The independent variables integrated two groups: sociodemographic, and related to the humanization of care. The positive evaluation (90.4\%) prevailed over the negative. Using Poisson regression, it was found that the negative evaluation was statistically associated with not having felt confidence in the dentist and staff, and not being able to talk to these professionals after the end of treatment. The results showed the preponderantly positive user evaluation of the service, and suggest that the evaluation may be more related to the humanization in services than to sociodemographic characteristics of the population.
\end{abstract}

Key words Health evaluation, Dental care, Humanization of assistance, Public health policies
Resumo Objetivou-se neste estudo conhecer a avaliação do usuário sobre o atendimento odontológico no Sistema Único de Saúde e analisar as associações entre a mesma, características sociodemográficas e aspectos relacionados à humanização nos serviços. Trata-se de pesquisa transversal com abordagem quantitativa, na qual 461 usuários responderam a entrevistas individuais. A variável desfecho foi obtida por meio da pergunta: "Como o(a) Senhor(a) considera o atendimento dado pelo dentista e pela equipe dele nessa unidade de saúde?". As respostas foram agrupadas nas categorias "avaliação positiva" e "avaliação negativa”. As variáveis independentes integraram dois grupos: sociodemográficas e relacionadas à humanização no atendimento. A avaliação positiva (90,4\%) prevaleceu sobre a negativa. Utilizandose a regressão de Poisson, verificou-se que a avaliação negativa esteve estatisticamente associada a não ter sentido confiança no dentista e na equipe, e a não ter facilidade para falar com esses profissionais após o fim do tratamento. Os resultados evidenciaram a preponderante avaliação positiva do usuário sobre o atendimento, e sugerem que a avaliação pode estar mais relacionada à humanização nos serviços do que às características sociodemográficas da população.

Palavras-chave Avaliação em saúde, Assistência odontológica, Humanização da assistência, Políticas públicas de saúde 


\section{Introdução}

O direito à saúde foi reconhecido internacionalmente em 1948 por meio da aprovação da Declaração Universal dos Direitos Humanos, pela Organização das Nações Unidas ${ }^{1,2}$. Seu texto afirma que todo ser humano tem direito a um padrão de vida capaz de assegurar a si e a sua família, a saúde e o bem estar². Em 1978, a Declaração de Alma-Ata ${ }^{3}$ reafirmou a saúde como um direito fundamental e estabeleceu a consecução de seu mais alto nível possível como a mais importante meta social mundial. No Brasil, a Constituição Federal de $1988^{4}$ e a lei $8.080 / 1990^{5}$ atribuíram ao Estado o dever de formular e de executar políticas com o objetivo de reduzir os riscos de doenças e de outros agravos, e de estabelecer condições que assegurem o acesso universal e igualitário às ações e aos serviços para a promoção, a proteção e a recuperação da saúde.

Apesar do Sistema Único de Saúde (SUS) ter incorporado o conceito de determinantes sociais da saúde ${ }^{5}$, a prática assistencial tradicional, historicamente adotada, estava centrada na exclusividade da dimensão biológica e na realização de procedimentos pressupondo que o indivíduo acometido por uma doença se mantivesse passivo diante das propostas de tratamento ${ }^{6,7}$. Havia a necessidade de se considerar as questões subjetivas envolvidas na prática de saúde, de humanizar o processo de trabalho. O termo humanização comporta uma gama de acepções ${ }^{8}$, mas pode ser entendido nos serviços de saúde, como a valorização dos diferentes sujeitos implicados no processo de produção de saúde: usuários, trabalhadores e gestores 9 . Antes mesmo da criação do SUS, o termo humanização já vinha sendo delineado no Brasil, recebendo influências e contribuições da Declaração Universal dos Direitos Humanos, da defesa em prol da assistência ao parto humanizado, da luta antimanicomial, e das demais críticas que se juntaram a estas, principalmente no que concerne ao modelo de assistência à saúde existente ${ }^{10}$. A partir dos anos 1990, a ideia de humanização passou a fazer parte do vocabulário da saúde com mais ênfase; inicialmente como um conjunto de princípios que criticavam o caráter impessoal e desumanizado da assistência à saúde, e que mais tarde foram traduzidos em diferentes propostas visando modificar as práticas assistenciais ${ }^{11}$.

O Ministério da Saúde entende que tem a responsabilidade de tornar a humanização um movimento capaz de fortalecer o SUS como política pública de saúde ${ }^{9}$. A partir desta visão, em 2003 foi criada a Política Nacional de Humanização
(PNH) da Atenção e Gestão no Sistema Único de Saúde, também chamada de HumanizaSUS ${ }^{12}$. A PNH surgiu num cenário de desafios, que exigia transformações no modelo de gestão e de atenção à saúde ${ }^{13}$, pois o SUS é ainda uma reforma incompleta, encontrando-se em pleno curso de mudanças ${ }^{9}$. Os princípios da $\mathrm{PNH}$ são a transversalidade; a indissociabilidade entre atenção e gestão; e o protagonismo, a corresponsabilidade e a autonomia dos sujeitos e dos coletivos ${ }^{9}$. Com a PNH, o tema da participação em saúde assumiu novos contornos, ampliando-se na direção da inclusão protagonista e corresponsável de todos os envolvidos no processo de produção de saúde ${ }^{12}$.

Dentre os dispositivos da PNH, estão os sistemas de escuta qualificada9 . A escuta do usuário significa, num primeiro momento, acolher todas as suas queixas ou relatos, mesmo quando possam parecer não interessar diretamente para o diagnóstico e tratamento ${ }^{6}$. O bom atendimento, baseado na escuta do usuário, e o bom desempenho profissional propiciam o vínculo do binômio usuário-serviço de saúde, otimizando o processo da assistência, e permitindo que os profissionais conheçam seus pacientes e as prioridades de cada $\mathrm{um}^{14}$. Muitas vezes, para o público, a forma do atendimento e a capacidade demonstrada pelos profissionais de saúde para compreender suas demandas e expectativas são fatores que chegam a ser mais valorizados do que a falta de médicos, a falta de espaço nos hospitais e a falta de medicamentos $^{15}$. Os indivíduos devem ser reconhecidos como sujeitos capazes de avaliar e intervir, modificando o próprio sistema e fortalecendo, assim, o fazer democrático da saúde ${ }^{16}$. Dessa forma, a avaliação do usuário pode contribuir para a redução dos problemas do cotidiano dos serviços de saúde e fomentar o surgimento de iniciativas de reorganização dos processos de trabalho que não demandem grandes investimentos financeiros ${ }^{17}$.

São escassos os trabalhos desenvolvidos a partir da avaliação do usuário, focando os aspectos humanísticos do atendimento ${ }^{18}$, sobretudo em odontologia. O mais recente levantamento epidemiológico de âmbito nacional na área de saúde bucal, conhecido como SB Brasil 2010, foi concluído em 2011 e apresentou dados sobre a situação da saúde bucal da população brasileira ${ }^{19}$. O instrumento utilizado para a coleta de dados no SB Brasil 2010 trouxe questões relevantes sobre condições socioeconômicas, utilização dos serviços odontológicos, morbidade bucal referida e autopercepção de saúde bucal, entretanto, questões relacionadas à humanização nos serviços odontológicos foram pouco abordadas ${ }^{19,20}$, 
refletindo uma importante lacuna existente nesse tema. Diante do exposto, o objetivo deste estudo foi conhecer a avaliação do usuário sobre o atendimento odontológico no SUS e analisar as associações entre essa avaliação, as características sociodemográficas e os aspectos relacionados à humanização nos serviços utilizados.

\section{Métodos}

Tratou-se de uma pesquisa transversal tipo inquérito, com abordagem quantitativa, que fez parte de um projeto multicêntrico. Foram incluídos 461 usuários do serviço odontológico do SUS, com idade mínima de 18 anos, que concordaram em participar da pesquisa, e que no dia da entrevista encontravam-se na área de acolhimento da unidade do SUS visitada, aguardando atendimento odontológico. Foram excluídos os menores de 18 anos, os portadores de necessidades especiais cognitivas, bem como aqueles cuja consulta odontológica seria a primeira na referida unidade.

A coleta de dados se deu por meio de entrevistas individuais realizadas por cirurgiões-dentistas (CD) devidamente calibrados, nas dezesseis unidades de saúde da zona urbana do município de Araçatuba, São Paulo, que dispunham de serviço de atendimento básico em odontologia. As entrevistas ocorreram no período de novembro de 2013 a maio de 2014, e foram distribuídas proporcionalmente entre as unidades de saúde, de acordo com a demanda atendida. O questionário estruturado aplicado foi adaptado a partir do modelo utilizado no Programa Nacional de Avaliação de Serviços de Saúde (PNASS) para pesquisa de satisfação do usuário ${ }^{21}$, e de instrumentos de avaliação externa do Programa da Melhoria do Acesso e da Qualidade (PMAQ) ${ }^{22,23}$. Foi realizado um estudo piloto para detecção de possíveis problemas e adequação do instrumento de coleta. Após a entrevista, cada usuário recebeu um manual impresso, elaborado especialmente para o estudo, contendo informações sobre os seus direitos e deveres perante o $\mathrm{SUS}^{24}$; e ainda um kit de saúde bucal, composto por escova, creme e fio dentais.

A variável desfecho foi a avaliação do usuário sobre o atendimento odontológico recebido, coletada por meio da pergunta: Como o(a) Senhor(a) considera o atendimento dado pelo dentista e pela equipe dele nessa unidade de saúde? A avaliação foi obtida em cinco níveis: muito bom, bom, regular, ruim e muito ruim. As respostas "muito bom" e "bom" foram agrupadas na categoria avaliação positiva do atendimento; e as respostas "regular", "ruim" e "muito ruim" foram agrupadas na categoria avaliação negativa. As variáveis independentes foram classificadas em dois grupos: sociodemográficas (gênero; faixa etária; ocupação; estado civil; escolaridade; renda familiar; e tipo de unidade de saúde onde é atendido), e relacionadas à humanização no atendimento (forma como é recebido no serviço odontológico; recebimento de informações sobre a condição de saúde bucal; êxito em conversar com o CD durante o atendimento; recebimento de informações sobre prevenção; interesse pela saúde bucal do usuário, demonstrado pelo CD e equipe; confiança no CD e equipe; ciência do nome do CD; e facilidade para falar com CD e equipe após a conclusão do tratamento).

Para o cálculo amostral, foi utilizada a prevalência de $50 \%$ para cada avaliação possível (positiva e negativa), no intuito de maximizar o tamanho da amostra. Ao total encontrado (384), foram acrescidos 20\% (77) para potencializar o efeito do estudo. Com isso, a amostra final foi de 461 indivíduos. O nível de confiança estipulado foi de 95\% para um erro amostral máximo de $5 \%$. Os dados foram processados com o auxílio do programa estatístico SPSS, versão $17.0^{25}$. Para verificar associações entre a variável desfecho e as independentes, foram realizadas análises bivariadas empregando os testes qui-quadrado e exato de Fisher, sendo este utilizado quando o primeiro mostrou-se inapropriado. Posteriormente, as variáveis associadas à avaliação do atendimento odontológico em nível de significância menor que $5 \%(\mathrm{p}<0,05)$ foram submetidas a análise multivariada. As magnitudes das associações foram obtidas pelas razões de prevalência, utilizando-se a regressão de Poisson, com nível de significância de 5\% e intervalos de confiança de 95\% (IC95\%).

Este estudo foi aprovado pelo Comitê de Ética em Pesquisa com seres humanos da Faculdade de Odontologia de Araçatuba - Universidade Estadual Paulista Júlio de Mesquita Filho (UNESP), tendo sido respeitados os ditames da resolução 466/12 do Conselho Nacional de Saúde (CNS).

\section{Resultados}

Dos 461 integrantes da amostra, um não respondeu à pergunta sobre avaliação do atendimento odontológico. Outras questões também apresentaram algumas não respostas, levando a diferen- 
ças no número absoluto de respondentes. Entre as características sociodemográficas, predominaram o gênero feminino (70,4\%), os empregados (44,3\%), os solteiros (57,0\%), a faixa etária de 18 a 29 anos (26,5\%), e a renda familiar menor que dois salários mínimos (67,9\%). Quanto à escolaridade, 40,8\% tinham apenas o ensino fundamental, e $48,7 \%$, o ensino médio. Dos participantes, $82,0 \%$ eram atendidos em uma das doze unidades de saúde integrantes da Estratégia Saúde da Família (ESF).

Quanto à avaliação do usuário sobre o atendimento odontológico recebido, 90,4\% dos entrevistados classificaram-no como muito bom ou bom (avaliação positiva) e 9,6\% como regular, ruim ou muito ruim (avaliação negativa).

Sobre as questões relacionadas à humanização no atendimento, 90,4\% disseram terem sido recebidos de uma forma boa ou muito boa no serviço de odontologia, 88,0\% receberam informações sobre a própria condição de saúde bucal, $74,3 \%$ conseguiram conversar com o CD nas vezes que precisaram, $67,0 \%$ sempre receberam informações sobre como prevenir as principais doenças da boca, e 93,0\% perceberam interesse do CD e da equipe por sua saúde bucal e seu tratamento. Além disso, 88,7\% dos entrevistados sentiram confiança no CD e na equipe, e $72,6 \%$ relataram facilidade para falar com o $C D$ e a equipe após a conclusão do tratamento. Apenas $36,7 \%$ sabiam o nome do CD que os atendia.

As Tabelas 1 e 2 trazem as análises bivariadas, por meio das quais se investigou as associações estatísticas entre avaliação do atendimento odontológico e as variáveis independentes. Nelas podem ser observadas pequenas diferenças no total de respondentes entre algumas variáveis, decorrentes das não respostas. Dentre as características sociodemográficas (Tabela 1), a escolaridade e o tipo de unidade de saúde onde o usuário era atendido apresentaram associação significativa $(\mathrm{p}=0,031$ e $\mathrm{p}=0,001$, respectivamente). Todas as variáveis relacionadas à humanização no atendimento odontológico (Tabela 2) estiveram associadas de forma significativa $(\mathrm{p}<0,001)$ à avaliação do atendimento.

A Tabela 3 traz os resultados da análise multivariada. Nela são apresentadas as razões de prevalência ajustadas da avaliação negativa do atendimento odontológico, segundo as variáveis independentes que haviam tido associação significativa nas análises bivariadas. A avaliação negativa do atendimento esteve estatisticamente associada a não ter sentido confiança no CD e equipe ( $R P=1,31$; IC 95\%: 1,17-1,48), e a não ter facilidade para falar com esses profissionais após a conclusão do tratamento $(\mathrm{RP}=1,11$; IC 95\%: 1,01-1,23). As demais variáveis não permaneceram associadas de forma significativa após a análise multivariada.

\section{Discussão}

Neste estudo, inicialmente, buscou-se conhecer a avaliação do usuário sobre o atendimento odontológico no SUS. O elevado percentual de avaliação positiva obtido corrobora os achados de outros estudos nacionais e internacionais sobre avaliação do atendimento em serviços de saúde, desenvolvidos a partir da visão do usuário $^{26-30}$. Essa alta avaliação positiva precisa ser vista com prudência. A possibilidade de que um bom trabalho venha sendo realizado nos serviços pesquisados e de que as entrevistas apenas tenham retratado tal situação, não deve ser ignorada, porém, outras considerações podem ajudar na compreensão desse resultado. Brandão et al. ${ }^{17}$ relatam que achados de alta satisfação podem estar relacionados a um nível baixo de expectativas, alcançando-se assim, mais facilmente, a avaliação positiva. Traverso-Yépez e Morais ${ }^{31}$ alertam para o equívoco do reconhecimento do serviço recebido como um favor ou doação, e não como um direito. Serapioni e Silva ${ }^{28}$ mencionam a supervalorização, por parte do usuário, do simples fato de ser atendido, sem se deter propriamente na avaliação do atendimento recebido.

$\mathrm{Na}$ literatura, alguns trabalhos identificaram associações entre condições sociodemográficas e a percepção que os sujeitos tiveram sobre os serviços de saúde $e^{32-34}$. No presente estudo, os entrevistados com grau de escolaridade superior apresentaram um percentual maior de avaliação negativa, quando comparados aos de graus médio e fundamental. Populações com baixos índices de escolaridade parecem ser mais condescendentes com os serviços de saúde prestados, avaliando-os mais positivamente ${ }^{17,29,35}$. Assim como a escolaridade, o tipo de unidade de saúde também se mostrou estatisticamente associado à avaliação do atendimento, na análise bivariada. Chamou atenção, a ausência de avaliação negativa entre aqueles atendidos em unidades não integrantes da ESF, contra $11,7 \%$ dessa avaliação entre os usuários de unidades com ESF. Esse achado talvez esteja relacionado ao fato de as equipes de trabalho (CD e auxiliares) das quatro unidades de saúde sem ESF visitadas serem formadas por ocupantes de cargos efetivos, e praticamente as 
Tabela 1. Associações entre avaliação do atendimento odontológico e características sociodemográficas. Araçatuba, São Paulo, 2013/2014.

\begin{tabular}{|c|c|c|c|c|c|c|c|}
\hline \multirow{3}{*}{ Variável } & \multicolumn{6}{|c|}{ Avaliação do atendimento } & \multirow{3}{*}{ Valor de $\mathrm{p}$} \\
\hline & \multicolumn{2}{|c|}{ Positiva } & \multicolumn{2}{|c|}{ Negativa } & \multicolumn{2}{|c|}{ Total } & \\
\hline & $\mathbf{n}$ & $\%$ & $\mathbf{n}$ & $\%$ & $\mathbf{n}$ & $\%$ & \\
\hline Gênero & & & & & & & 0,165 \\
\hline Masculino & 119 & 87,5 & 17 & 12,5 & 136 & 100,0 & \\
\hline Feminino & 297 & 91,7 & 27 & 8,3 & 324 & 100,0 & \\
\hline Faixa etária & & & & & & & 0,386 \\
\hline 18 a 29 & 107 & 87,7 & 15 & 12,3 & 122 & 100,0 & \\
\hline 30 a 39 & 87 & 90,6 & 9 & 9,4 & 96 & 100,0 & \\
\hline 40 a 49 & 103 & 94,5 & 6 & 5,5 & 109 & 100,0 & \\
\hline 50 a 59 & 66 & 91,7 & 6 & 8,3 & 72 & 100,0 & \\
\hline 60 ou mais & 53 & 86,9 & 8 & 13,1 & 61 & 100,0 & \\
\hline Ocupação & & & & & & & 0,785 \\
\hline Empregado & 188 & 92,2 & 16 & 7,8 & 204 & 100,0 & \\
\hline Autônomo & 60 & 88,2 & 8 & 11,8 & 68 & 100,0 & \\
\hline Aposentado & 40 & 93,0 & 3 & 7,0 & 43 & 100,0 & \\
\hline Dona de casa & 75 & 89,3 & 9 & 10,7 & 84 & 100,0 & \\
\hline Estudante & 34 & 87,2 & 5 & 12,8 & 39 & 100,0 & \\
\hline Desempregado & 19 & 86,4 & 3 & 13,6 & 22 & 100,0 & \\
\hline Estado civil & & & & & & & 0,679 \\
\hline Casado/União estável & 127 & 88,8 & 16 & 11,2 & 143 & 100,0 & \\
\hline Solteiro & 237 & 90,5 & 25 & 9,5 & 262 & 100,0 & \\
\hline Separado/Divorciado & 18 & 94,7 & 1 & 5,3 & 19 & 100,0 & \\
\hline Viúvo & 34 & 94,4 & 2 & 5,6 & 36 & 100,0 & \\
\hline Escolaridade & & & & & & & 0,031 \\
\hline Superior & 39 & 81,3 & 9 & 18,8 & 48 & 100,0 & \\
\hline Médio & 208 & 93,3 & 15 & 6,7 & 223 & 100,0 & \\
\hline Fundamental & 168 & 89,8 & 19 & 10,2 & 187 & 100,0 & \\
\hline Renda familiar & & & & & & & 0,863 \\
\hline$<2$ salários mínimos & 273 & 90,4 & 29 & 9,6 & 302 & 100,0 & \\
\hline$\geq 2$ salários mínimos & 130 & 90,9 & 13 & 9,1 & 143 & 100,0 & \\
\hline Tipo de unidade de saúde & & & & & & & 0,001 \\
\hline Com ESF & 333 & 88,3 & 44 & 11,7 & 377 & 100,0 & \\
\hline Sem ESF & 83 & 100,0 & 0 & 0,0 & 83 & 100,0 & \\
\hline
\end{tabular}

As diferenças no número de respondentes entre algumas variáveis correspondem às não respostas.

mesmas há anos, tendo assim, desenvolvido vínculos fortes e permanentes com a comunidade. O acompanhamento ao longo do tempo oferece ao usuário um cuidado mais adequado ${ }^{16}$. Já nas demais unidades, com ESF, observou-se durante as visitas, que a imensa maioria dos profissionais era contratada por tempo determinado, mediante terceirização, gerando certa rotatividade nos membros das equipes, e dificultando o estabelecimento dos vínculos necessários. Segundo levantado por Serapioni e Silva ${ }^{28}$, a boa relação entre profissionais de saúde e usuários contribui para a constituição desses vínculos, que por sua vez favorecem a inserção das equipes no ambiente de vida dos pacientes.
Observando-se ainda os resultados das análises bivariadas, vê-se que a relação interpessoal com a equipe odontológica parece ter tido participação direta no julgamento do atendimento recebido, pois todas as variáveis relacionadas à humanização apresentaram associação estatisticamente significativa com a avaliação do atendimento. Rahmqvist e Bara ${ }^{34}$ também encontraram associação entre a forma como o usuário percebe o serviço de saúde, e as relações de intersubjetividade com os profissionais. O usuário, em geral, ao avaliar o cuidado prestado, reconhece quando os profissionais demonstram respeito, atenção, elo de afetividade, confiança e credibilidade ${ }^{36}$. Nesta pesquisa, entre aqueles que foram bem recebi- 
Tabela 2. Associações entre avaliação do atendimento odontológico e variáveis relacionadas à humanização. Araçatuba, São Paulo, 2013/2014.

\begin{tabular}{|c|c|c|c|c|c|c|c|}
\hline \multirow{3}{*}{ Variável } & \multicolumn{6}{|c|}{ Avaliação do atendimento } & \multirow{3}{*}{ Valor de $\mathrm{p}$} \\
\hline & \multicolumn{2}{|c|}{ Positiva } & \multicolumn{2}{|c|}{ Negativa } & \multicolumn{2}{|c|}{ Total } & \\
\hline & $\mathbf{n}$ & $\%$ & $\mathbf{n}$ & $\%$ & $\mathbf{n}$ & $\%$ & \\
\hline Forma como é recebido no serviço odontológico & & & & & & & $<0,001$ \\
\hline Boa/muito boa & 392 & 94,2 & 24 & 5,8 & 416 & 100,0 & \\
\hline Regular/ruim/muito ruim & 24 & 54,5 & 20 & 45,5 & 44 & 100,0 & \\
\hline $\begin{array}{l}\text { Recebimento de informações sobre a condição } \\
\text { de saúde bucal }\end{array}$ & & & & & & & $<0,001$ \\
\hline Sim & 381 & 94,8 & 21 & 5,2 & 402 & 100,0 & \\
\hline Não & 34 & 61,8 & 21 & 38,2 & 55 & 100,0 & \\
\hline $\begin{array}{l}\text { Exito em conversar com o CD durante o } \\
\text { atendimento }\end{array}$ & & & & & & & $<0,001$ \\
\hline Sim, sempre & 328 & 96,2 & 13 & 3,8 & 341 & 100,0 & \\
\hline Sim, algumas vezes & 21 & 72,4 & 8 & 27,6 & 29 & 100,0 & \\
\hline Não & 24 & 58,5 & 17 & 41,5 & 41 & 100,0 & \\
\hline Nunca precisou & 42 & 87,5 & 6 & 12.5 & 48 & 100,0 & \\
\hline Recebimento de informações sobre prevenção & & & & & & & $<0,001$ \\
\hline Sim, sempre & 296 & 97,7 & 7 & 2,3 & 303 & 100,0 & \\
\hline Sim, algumas vezes & 45 & 81,8 & 10 & 18,2 & 55 & 100,0 & \\
\hline Não & 67 & 71,3 & 27 & 28,7 & 94 & 100,0 & \\
\hline $\begin{array}{l}\text { Interesse pela saúde bucal do usuário, } \\
\text { demonstrado pelo CD e equipe }\end{array}$ & & & & & & & $<0,001$ \\
\hline $\operatorname{Sim}$ & 406 & 94,9 & 22 & 5,1 & 428 & 100,0 & \\
\hline Não & 10 & 31,3 & 22 & 68,7 & 32 & 100,0 & \\
\hline Confiança no CD e equipe & & & & & & & $<0,001$ \\
\hline Sim & 395 & 97,1 & 12 & 2,9 & 407 & 100,0 & \\
\hline Não & 20 & 38,5 & 32 & 61,5 & 52 & 100,0 & \\
\hline Ciência do nome do CD & & & & & & & $<0,001$ \\
\hline $\operatorname{Sim}$ & 163 & 96,4 & 6 & 3,6 & 169 & 100,0 & \\
\hline Não & 253 & 86,9 & 38 & 13,1 & 291 & 100,0 & \\
\hline $\begin{array}{l}\text { Facilidade para falar com CD e equipe após a } \\
\text { conclusão do tratamento }\end{array}$ & & & & & & & $<0,001$ \\
\hline Sim & 310 & 97,5 & 8 & 2,5 & 318 & 100,0 & \\
\hline Não & 20 & 46,5 & 23 & 53,5 & 43 & 100,0 & \\
\hline Nunca precisou & 70 & 90,9 & 7 & 9,1 & 77 & 100,0 & \\
\hline
\end{tabular}

As diferenças no número de respondentes entre algumas variáveis correspondem às não respostas.

Tabela 3. Razões de prevalência ajustadas da avaliação negativa do atendimento odontológico, segundo variáveis independentes. Araçatuba, São Paulo, 2013/2014.

\begin{tabular}{|c|c|c|c|c|c|}
\hline \multirow{2}{*}{ Variável } & \multicolumn{2}{|c|}{ Avaliação negativa } & \multirow{2}{*}{$\begin{array}{c}\text { RP } \\
\text { Ajustada }\end{array}$} & \multirow{2}{*}{ (IC 95\%) } & \multirow{2}{*}{$\begin{array}{l}\text { Valor } \\
\text { de p }\end{array}$} \\
\hline & $\mathbf{n}$ & $\%$ & & & \\
\hline \multicolumn{6}{|l|}{ Confiança no CD e equipe } \\
\hline Sim & 12 & 2,9 & 1,00 & & \\
\hline Não & 32 & 61,5 & 1,31 & $1,17-1,48$ & $<0,001$ \\
\hline \multicolumn{6}{|c|}{$\begin{array}{l}\text { Facilidade para falar com CD e equipe após a } \\
\text { conclusão do tratamento }\end{array}$} \\
\hline $\operatorname{Sim}$ & 8 & 2,5 & 1,00 & & \\
\hline Não & 23 & 53,5 & 1,11 & $1,01-1,23$ & 0,026 \\
\hline Nunca precisou & 7 & 9,1 & 1,00 & $0,95-1,05$ & 0,900 \\
\hline
\end{tabular}

A diferença no número de respondentes entre as variáveis corresponde às não respostas. 
dos no serviço odontológico; que foram informados sobre a própria condição de saúde bucal; que conseguiram conversar com o CD durante $\mathrm{o}$ atendimento; que sempre receberam informações sobre prevenção de doenças bucais; que perceberam interesse do CD e equipe pela sua saúde bucal; que sentiram confiança no $\mathrm{CD}$ e equipe; que sabiam o nome do $\mathrm{CD}$; e que tiveram facilidade para falar com o CD e equipe após a conclusão do tratamento; houve no mínimo 94,2\% de avaliações positivas. Entretanto, a motivação para adoção de condutas humanizadas por profissionais de saúde bucal, deve ir muito além da busca por boas avaliações dos serviços ofertados. Usuários entrevistados por Reis et al. ${ }^{37}$ em uma pesquisa qualitativa, expuseram a importância da relação entre profissionais e pacientes, e sua influência sobre o resultado do tratamento.

Também no contexto da relação profissional-usuário, um dado mostrou-se discrepante do que se esperaria para o cenário encontrado. Apesar dos resultados terem indicado a ocorrência frequente de diálogo com os membros da equipe, $63,3 \%$ dos participantes da pesquisa não sabiam o nome do CD, e destes, $86,9 \%$ avaliaram $\mathrm{o}$ atendimento positivamente. Como as respostas às questões sobre humanização apontaram para a presença de um ótimo relacionamento com a equipe odontológica, seria esperado que o usuário soubesse o nome do CD que o atendeu. Novamente, o baixo nível de expectativas relatado por Brandão et al. ${ }^{17}$ pode ajudar a compreender tal circunstância, porém, sem desconsiderar a existência de condutas humanizadas que possam justificar, em parte, a aprovação encontrada. Outro fato que pode contribuir para a interpretação dessa realidade é a já referida rotatividade nos membros das equipes de trabalho de algumas unidades pesquisadas. A realização de grupos focais ou outra técnica de pesquisa qualitativa seria apropriada para o aprofundamento da discussão sobre esse achado em futuros trabalhos.

Diante da predominância da avaliação positiva do atendimento odontológico, buscou-se, na análise multivariada, conhecer como e quais variáveis independentes estariam associadas à reduzida avaliação negativa encontrada, além da força dessas associações. Não confiar no CD e equipe foi a condição mais fortemente associada à avaliação negativa do atendimento recebido, seguida de não ter facilidade para falar com o CD e equipe após a conclusão do tratamento. Considerando que o medo e a ansiedade são sentimentos comuns a pacientes que necessitam de tratamento odontológico $^{38}$; não confiar no CD e equipe pode dificultar a superação desses sentimentos, e colaborar para a avaliação negativa da experiência vivida dentro do consultório. Da mesma forma, buscar esses profissionais para esclarecer dúvidas surgidas após o fim do tratamento e encontrar dificuldades para ser ouvido é uma situação frustrante e causadora potencial de insatisfação. Ter a habilidade de escutar, explicar e dar atenção ao usuário contribui para a superação de barreiras e fortalecimento de vínculos. Assim como neste estudo, a importância da humanização no atendimento também se apresentou de maneira muito incisiva em um trabalho onde, por meio de questões abertas, $43 \%$ dos usuários disseram que o que mais valorizavam na ESF era a boa relação com os profissionais; e 36\% externaram que o que mais prejudicava a ESF era a maneira de atender dos mesmos ${ }^{28}$.

A subjetividade e a multidimensionalidade que envolvem a avaliação de serviços de saúde podem ter exercido papel importante sobre os resultados deste estudo, contribuindo para a ocorrência do elevado percentual de avaliação positiva. Talvez essa acentuada positividade tenha concorrido para a não permanência, após a análise multivariada, de algumas associações estatísticas verificadas nas análises bivariadas. Mesmo assim, é importante verificar que a avaliação negativa foi mais frequente entre aqueles que, de forma geral, não perceberam a existência de ações humanizadas durante o atendimento.

Novos trabalhos são necessários para aprofundar as discussões sobre o tema e fornecer o máximo possível de subsídios para que usuários, profissionais e gestores de saúde possam, coletivamente, consolidar a humanização como política pública permanente e indispensável nos serviços de saúde bucal e no SUS.

\section{Conclusão}

Essa pesquisa evidenciou a preponderante avaliação positiva que o usuário do SUS teve sobre o atendimento odontológico, assim como a relação existente entre avaliação e humanização no atendimento. Os resultados sugerem que a avaliação pode estar mais relacionada a aspectos da humanização nos serviços do que a características sociodemográficas da população. Todavia, a obtenção de boas avaliações deve ser consequência de todo o processo de trabalho, inclusive de sua humanização, e não uma finalidade em si mesmo. 


\section{Colaboradores}

SAS Moimaz participou da concepção e delineamento da pesquisa, e da elaboração do artigo. AMC Lima participou do delineamento da pesquisa, da coleta de dados e da elaboração do artigo. CAS Garbin e NA Saliba participaram da concepção e do delineamento da pesquisa. JE Corrente trabalhou na análise dos dados. Ademais, todos os autores participaram da interpretação dos dados, da revisão crítica do conteúdo intelectual, e da aprovação da versão final a ser publicada.

\section{Agradecimentos}

Ao Conselho Nacional de Desenvolvimento Científico e Tecnológico (CNPq) pelo auxílio financeiro.

\section{Referências}

1. Mitre SM, Andrade EIG, Cotta RMM. Avanços e desafios do acolhimento na operacionalização e qualificação do Sistema Único de Saúde na Atenção Primária: um resgate da produção bibliográfica do Brasil. Cien Saude Colet 2012; 17(8):2071:2085.

2. Organização das Nações Unidas (ONU). Assembleia Geral. Declaração Universal dos Direitos Humanos. Paris: ONU; 1948. [acessado 2014 nov 20]. Disponível em: http://unesdoc.unesco.org/images/0013/001394/ 139423 por.pdf.

3. Organização Mundial de Saúde (OMS). Declaração de Alma-Ata. Alma-Ata: OMS; 1978. [acessado 2014 nov 20]. Disponível em: http://bioeticaediplomacia.org/ wp-content/uploads/2013/10/alma-ata.pdf.

4. Brasil. Constituição da República Federativa do Brasil. Brasília: Senado; 1988.

5. Brasil. Lei no 8.080, de 19 de setembro de 1990. Dispõe sobre as condições para a promoção, proteção e recuperação da saúde, a organização e o funcionamento dos serviços correspondentes e dá outras providências. Diário Oficial da União 1990; 20 set.

6. Brasil. Ministério da Saúde (MS). Secretaria de Atenção à Saúde. Política Nacional de Humanização da Atenção e Gestão do SUS: clínica ampliada e compartilhada. Brasília: MS; 2009.

7. Brasil. Ministério da Saúde (MS). Secretaria de Atenção à Saúde. Núcleo Técnico da Política Nacional de Humanização. Acolhimento nas práticas de produção de saúde. 2a ed. Brasília: MS; 2010.

8. Lima CC, Guzman SM, Benedetto MAC, Gallian DMC Humanidades e humanização em saúde: a literatura como elemento humanizador para graduandos da área de saúde. Interface (Botucatu) 2014; 18(48):139-150.

9. Brasil. Ministério da Saúde (MS). Secretaria de Atenção à Saúde. Núcleo Técnico da Política Nacional de Humanização. HumanizaSUS: documento base para gestores e trabalhadores do SUS. 4a ed. Brasília: MS; 2012.

10. Conceição TS. A Política Nacional de Humanização e suas implicações para a mudança do modelo de atenção e gestão na saúde: notas preliminares. SER Soc 2009; 11(25):194-220.

11. Vaitsman J, Andrade GRB. Satisfação e responsividade: formas de medir a qualidade e a humanização da assistência à saúde. Cien Saude Colet 2005; 10(3):599-613.

12. Pasche DF, Passos E, Hennington EA. Cinco anos da Política Nacional de Humanização: trajetória de uma política pública. Cien Saude Colet 2011; 16(11):45414548.

13. Hennington EA. Gestão dos processos de trabalho e humanização em saúde: reflexões a partir da ergologia. Rev Saude Publica 2008; 42(3):555-561.

14. Moimaz SAS, Marques JAM, Saliba O, Garbin CAS, Zina LG, Saliba NA. Satisfação e percepção do usuário do SUS sobre o serviço público de saúde. Physis 2010; 20(4):1419-1440.

15. Brasil. Ministério da Saúde (MS). Secretaria de Atenção à Saúde. Programa Nacional de Humanização da Assistência Hospitalar. Brasília: MS; 2001. 
16. Moraes VD, Campos CEA, Brandão AL. Estudo sobre dimensões da avaliação da Estratégia Saúde da Família pela perspectiva do usuário. Physis 2014; 24(1):127146.

17. Brandão ALRBS, Giovanella L, Campos CEA. Avaliação da atenção básica pela perspectiva dos usuários: adaptação do instrumento EUROPEP para grandes centros urbanos brasileiros. Cien Saude Colet 2013; 18(1):103114.

18. Lima TJV, Arcieri RM, Garbin CAS, Moimaz SAS, Saliba O. Humanização na atenção básica de saúde na percepção de idosos. Saude Soc 2014; 23(1):265-276.

19. Brasil. Ministério da Saúde (MS). Secretaria de Atenção à Saúde. Departamento de Atenção Básica. Coordenação Nacional de Saúde Bucal. SB Brasil 2010 - Pesquisa Nacional de Saúde Bucal: projeto técnico. Brasília: MS; 2009.

20. Brasil. Ministério da Saúde (MS). Secretaria de Atenção à Saúde. Departamento de Atenção Básica. Coordenação Geral de Saúde Bucal. SB Brasil 2010 - Pesquisa Nacional de Saúde Bucal: resultados principais. Brasília: MS; 2011.

21. Brasil. Ministério da Saúde (MS). Secretaria de Atenção à Saúde. Departamento de Regulação Avaliação e Controle de Sistemas. Coordenação Geral de Regulação e Avaliação. Caderno do Programa Nacional de Avaliação dos Serviços de Saúde - PNASS: edição 2004-2005. Brasília: MS; 2004.

22. Brasil. Ministério da Saúde (MS). Secretaria de Atenção à Saúde. Departamento de Atenção Básica. Programa de Melhoria do Acesso e da Qualidade - PMAQ: instrumento de avaliação externa para as equipes de atenção básica. Brasília: MS; 2013.

23. Brasil. Ministério da Saúde (MS). Secretaria de Atenção à Saúde. Departamento de Atenção Básica. Programa de Melhoria do Acesso e da Qualidade - PMAQ: instrumento de avaliação externa para as equipes de saúde bucal. Brasília: MS; 2013.

24. Moimaz SAS, Fadel CB, Bordin D, Rovida TAS, Saliba NA. Cidadania e saúde bucal. Araçatuba: UNESP, UEPG; 2013.

25. SPSS Inc. Released. SPSS Statistics for Windows. [computer program]. Version 17.0. Chicago: SPSS Inc.; 2008.

26. Castro HCO, Machado LZ, Walter MIMT, Ranincheski SM, Schmidt BV, Marinho DNC, Campos TMA. A satisfação dos usuários com o Sistema Único de Saúde (SUS). Soc Debate 2008; 14(2):113-134.

27. Zils AA, Castro RCL, Oliveira MMC, Harzheim E, Duncan BB. Satisfação dos usuários da rede de Atenção Primária de Porto Alegre. Rev Bras Med Fam Comunidade 2009; 4(16):270-276.

28. Serapioni M, Silva MGC. Avaliação da qualidade do Programa Saúde da Família em municípios do Ceará. Uma abordagem multidimensional. Cien Saude Colet 2011; 16(11):4315-4326.
29. Bjertnaes OA, Sjetne IS, Iversen HH. Overall patient satisfaction with hospitals: effects of patient-reported experiences and fulfilment of expectations. BMJ Qual Saf 2012; 21(1):39-46.

30. Al-Haboubi M, Klass C, Jones K, Bernabé E, Gallagher JE. Inequalities in the use of dental services among adults in inner South East London. Eur J Oral Sci 2013; 121(3):176-181.

31. Traverso-Yépez M, Morais NA. Reivindicando a subjetividade dos usuários da Rede Básica de Saúde: para uma humanização do atendimento. Cad Saude Publica 2004; 20(1):80-88

32. Priporas C, Laspa C, Kamenidou I. Patient satisfaction measurement for in-hospital services: a pilot study in Greece. J Med Marketing 2008; 8(4):325-340.

33. Gouveia GC, Souza WV, Luna CF, Souza-Júnior PRB, Szwarcwald CL. Satisfação dos usuários do sistema de saúde brasileiro: fatores associados e diferenças regionais. Rev Bras Epidemiol 2009; 12(3):281-296.

34. Rahmqvist M, Bara A. Patient characteristics and quality dimensions related to patient satisfaction. Int J Qual Health Care 2010; 22(2):86-92.

35. Santiago RF, Mendes ACG, Miranda GMD, Duarte PO, Furtado BMASM, Souza WV. Qualidade do atendimento nas Unidades de Saúde da Família no município de Recife: a percepção do usuário. Cien Saude Colet 2013; 18(1):35-44.

36. Schimith MD, Simon BS, Brêtas ACP, Budó MLD. Relações entre profissionais de saúde e usuários durante as práticas em saúde. Trab Educ Saude 2011; 9(3):479-503.

37. Reis C, Martelli-Júnior H, Franco BM, Santos AA, Ramalho LMP. Avaliação do serviço de saúde bucal no município de Grão Mogol, Minas Gerais, Brasil: "a voz do usuário". Cien Saude Colet 2009; 14(4):1287-1295.

38. Medeiros LA, Ramiro FMS, Lima CAA, Souza LMA, Fortes TMV, Groppo FC. Avaliação do grau de ansiedade dos pacientes antes de cirurgias orais menores. Rev Odontol UNESP 2013; 42(5):357-363.

Artigo apresentado em 13/07/2015

Aprovado em 06/09/2015

Versão final apresentada em 08/09/2015 